\title{
HELICOPTER FLIGHT SIMULATION BASED ON URANS SOLVER AND VIRTUAL BLADE MODEL
}

\author{
Wieńczysław Stalewski, Katarzyna Surmacz \\ Eukasiewicz Research Network - Institute of Aviation \\ Krakowska Av. 110/114, 02-256 Warsaw, Poland \\ e-mail:wienczyslaw.stalewski@ilot.edu.pl,katarzyna.surmacz@ilot.edu.pl
}

\begin{abstract}
The methodology of simulation of a rotorcraft flight has been developed and applied to simulate several stages of flight of light helicopter. The methodology is based on coupling of several computational models of Computational Fluid Dynamics, Flight Dynamic. The essence of the methodology consists in calculation of aerodynamic forces acting on the flying rotorcraft by solving during the simulation the Unsteady Reynolds-Averaged Navier-Stokes (URANS) equations. In this approach, the rotorcraft is flying inside the computational $3 D$ mesh modelling the space filled with the air. The flight simulation procedure is completely embedded in the URANS solver ANSYS FLUENT. Flow effects caused by rotating blades of main or tail rotor are modelled by application of the developed Virtual Blade Model (VBM). In this approach, real rotors are replaced by volume discs influencing the flow field similarly as rotating blades. Time-averaged aerodynamic effects of rotating blades are modelled using momentum source terms placed inside the volume-disc zones. The momentum sources are evaluated based on the Blade Element Theory, which associates local flow parameters in the blade sections with databases of 2D-aerodynamic characteristics of these sections. Apart of the VBM module, two additional UDF modules support the simulation of helicopter flight: the module responsible for modelling of all kinematic aspects of the flight and the module gathering the momentary aerodynamic loads and solves 6 DOFEquations describing a motion of the helicopter seen as solid body. Exemplary simulation of helicopter flight, starting from a hover, through an acceleration and fast flight until a deceleration and steep descent, has been discussed.
\end{abstract}

Keywords: helicopter flight simulation, URANS, Virtual Blade Model

\section{Introduction}

The methodology discussed below, developed and implemented independently by the Authors, allows simulation of selected rotorcraft flight conditions without the need for laborious development of extensive databases containing aerodynamic characteristics of its individual components (main rotor, fuselage, tail rotor, control surfaces, etc.). The assumption of the methodology was to enable, among others, simulations of transient flight (also) close to the ground, e.g. during take-off or landing. The development of such a methodology was motivated by the needs of research and design works on new rotorcrafts, under which the assessment of performance, controllability, and manoeuvrability of these constructions was an important problem. This especially concerns the reliable assessment of a helicopter behaviour in dangerous flight conditions like emergency landing in autorotation or safe escape from the Vortex Ring State. To carry out these challenging tasks, a computational methodology was needed, which on the one hand would reliably reproduce most aspects of the modelled physical phenomena, and on the other would create the possibility of simulating unconstrained flight of rotorcraft in an acceptable time of calculations, performed on available, limited hardware resources. These challenges were to be met by the original computational methodology developed by the Authors.

\section{Methodology}

The general scheme of the developed and implemented by the Authors methodology of rotorcraft flight simulation [3] is presented in Fig. 1. The methodology discussed is based on three assumptions: 


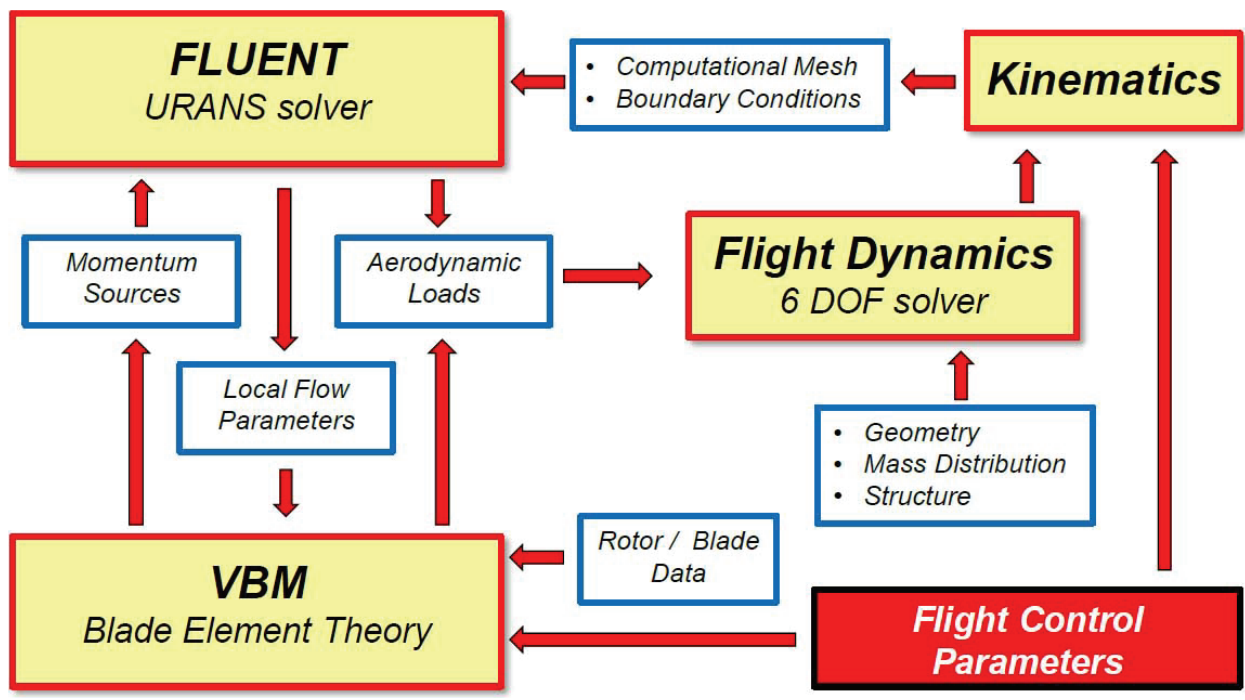

Fig. 1. General scheme of the developed methodology of rotorcraft-flight simulation [3]

- the aerodynamic model of the flight is based on the solution of URANS (Unsteady Reynolds Averaged Navier-Stokes) equations describing the air flow around the flying rotorcraft. In particular, all aerodynamic forces acting on fixed and non-rotating elements (fuselage, tail, landing gear, etc.) are calculated using this approach. URANS equations are solved by the ANSYS FLUENT solver [1],

- aerodynamic forces generated by rotating lifting-surface systems (main rotor, tail rotor, propeller, etc.) and flow effects induced by these systems are determined using the Virtual Blade Model module coupled with the ANSYS FLUENT code,

- simulation of a transient flight of the rotorcraft refers to the motion of the entire aircraft treated as a rigid body and is performed in relation to the time scale associated with this motion. The helicopter motion is modelled based on a system of equations describing the movement of a rigid body with 6 degrees of freedom. The FLIGHT-DYNAMICS module is responsible for the solution of this system, while all activities related to rotorcraft kinematics are carried out by the KINEMATICS module.

The rotorcraft flight simulation procedure is embedded in the URANS solver ANSYS FLUENT. Flow effects caused by rotating lifting surfaces are modelled by application of the developed UDF (User Defined Function) module Virtual Blade Model (VBM) [2], which is compiled and linked with the essential code of the ANSYS FLUENT software. In the VBM approach, real rotors are replaced by volume discs influencing the flow field in a manner similar to real rotating blades. Time-averaged aerodynamic effects of rotating lifting surfaces are modelled by means of artificial momentum sources placed inside the volume-disc zones localised in regions of activity of real rotors. Such zones, replacing the real main and tail rotor are shown in Fig. 2 . The intensities of momentum sources are evaluated based on the Blade Element Theory, associating local flow parameters (density and velocity) in rotor-disc zones with aerodynamic characteristics of blade airfoils. Data bases of these characteristics (in general: lift and drag coefficients as functions of angle of attack, for several sets of Mach and Reynolds numbers, similar to those that are expected on the real rotor blades) should be prepared before starting the flight simulation. The original VBM code was significantly modified and expanded by the Authors of this article.

The computational model of the light helicopter used in the presented research as a technology demonstrator, is shown in Fig. 2. In general, this model includes all principal components of the helicopter: fuselage, horizontal stabilizer, vertical tail, main rotor, and tail rotor that are rotating inside the fenestron. Taking into account the assumed simplified modelling of the effects of rotating lifting surfaces, geometries of main rotor and tail rotor were replaced by cylindrical fluid zones, in a manner typical for the VBM methodology, as shown in Fig. 2. 


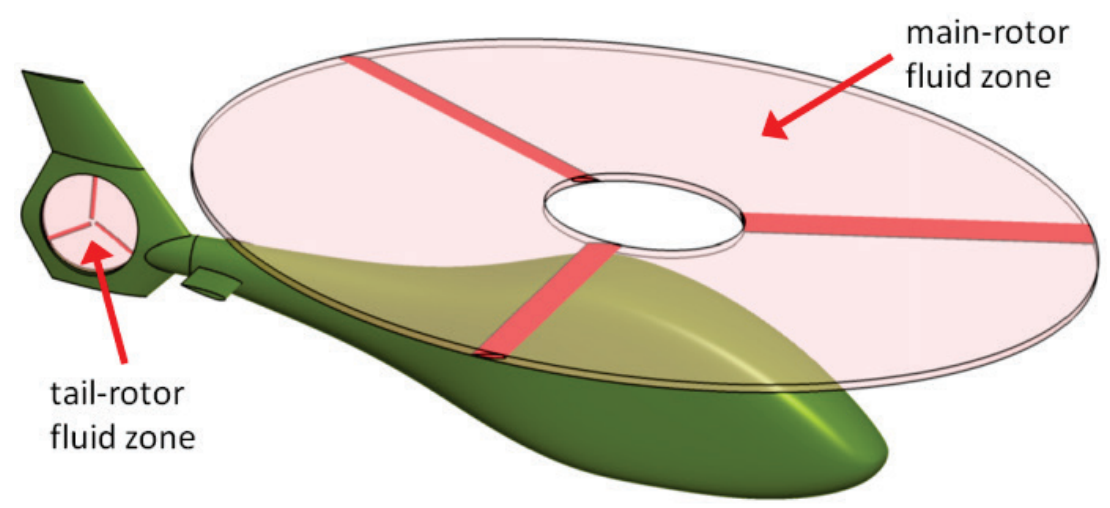

Fig. 2. Computational model of the helicopter [2]

All URANS simulations discussed in this article were conducted using the ANSYS FLUENT solver with the Spalart-Allmaras turbulence model. In these simulations, the air stayed motionless while the flight of the helicopter was modelled by the use of Moving Reference Frame Method, implemented in ANSYS FLUENT.

\section{Results of simulation of flight of a helicopter}

Presented below the helicopter flight consisted of the following six phases:

- Phase No. 1: Hover (without ground effect),

- Phase No. 2: Transition to horizontal flight,

- Phase No. 3: Acceleration of the helicopter,

- Phase No. 4: Fast horizontal flight,

- Phase No. 5: Deceleration of the helicopter,

- Phase No. 6: Steep descent.

Results of simulation of described above flight of the helicopter are presented in:

- Fig. 3 - velocity-magnitude contours around the helicopter in sequential phases (1-6) of flight,

- Fig. 4 - streamlines flowing from the main and tail rotor, in sequential phases (1-6) of flight,

- Fig. 5 - velocity-magnitude contours on the main-rotor disc and streamlines flowing from the main and tail rotor in sequential phases (1-6) of flight.

In the Flight Phase No. 1, the helicopter main rotor was positioned horizontally to the ground. Both the velocity-magnitude contours and streamlines showed the flow field typical for a hover.

In the Flight Phase No. 2, the helicopter was tilted by 9 degrees nose down, to start a horizontal motion.

In the Flight Phase No. 3, the helicopter reached the flight velocity $10 \mathrm{~m} / \mathrm{s}$ and it continued the horizontal acceleration.

In the Flight Phase No 4, the helicopter continued the fast horizontal flight at a speed of $40 \mathrm{~m} / \mathrm{s}$.

In the Flight Phase No. 5, the helicopter was tilted by 10 degrees nose up, to decelerate the horizontal velocity.

In the Flight Phase No. 6, the helicopter performed steep descent at angle 60 degrees to the ground. The flight velocity in this phase was approximately $8 \mathrm{~m} / \mathrm{s}$. One can notice that in these flight conditions a dangerous phenomenon - the Vortex Ring State, occurred.

\section{Conclusions}

The efficient and reliable methodology of computational simulation of non-stationary flight of a helicopter has been developed and implemented. The methodology is based on the solution of URANS equations in a domain surrounding the flying helicopter. Instead of true modelling of 
rotor blades, the aerodynamic forces generated by rotating lifting-surface systems (main rotor, tail rotor, propeller, etc.) and flow, effects induced by these systems are determined using the Virtual Blade Model. Simulation of a transient flight of the helicopter refers to the motion of reference frame connected with the rotorcraft treated as a rigid body and is performed in relation to the time scale associated with this motion. The rotorcraft motion is described by a system of equations describing the movement of a rigid body with 6 degrees of freedom.

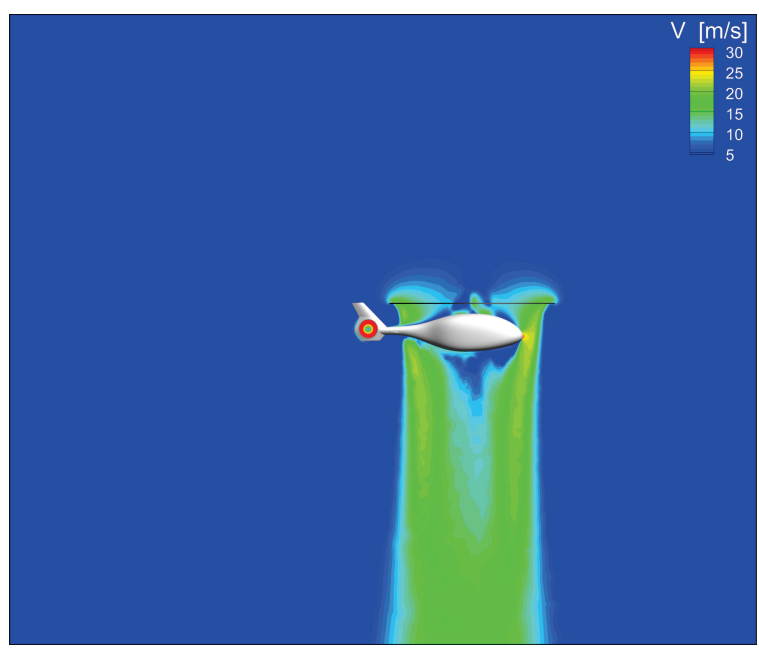

phase 1

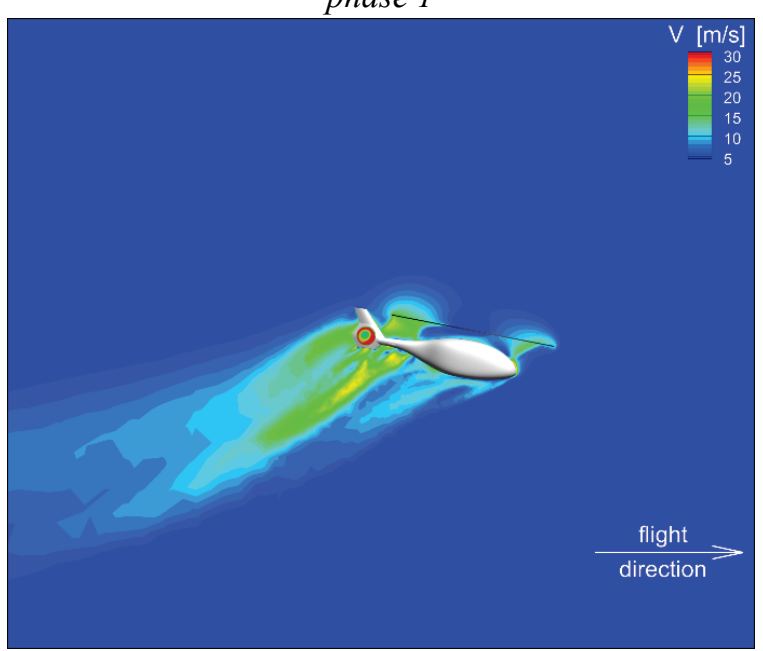

phase 3

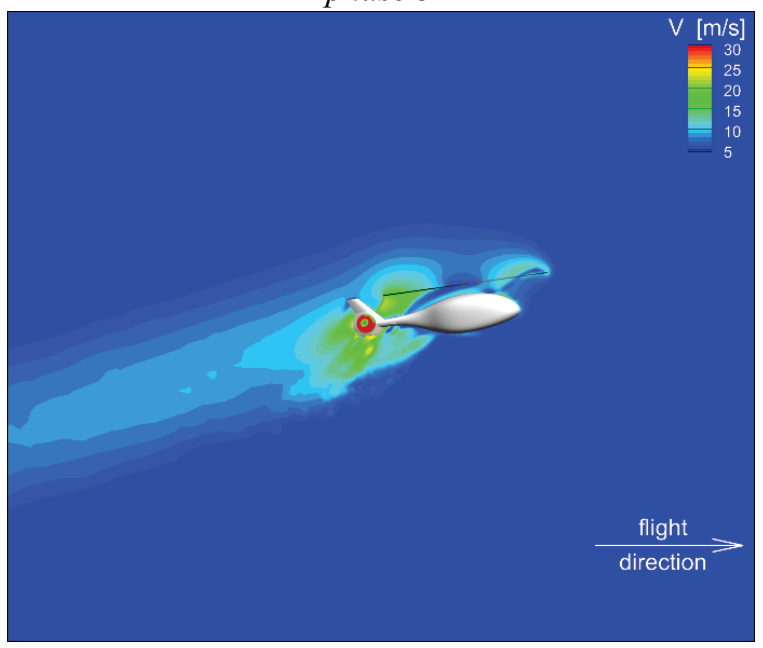

phase 5

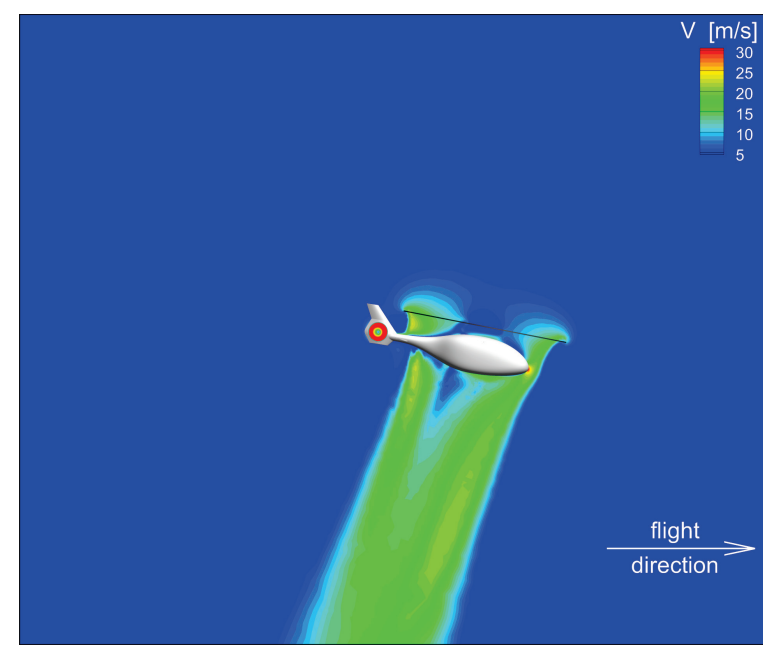

phase 2

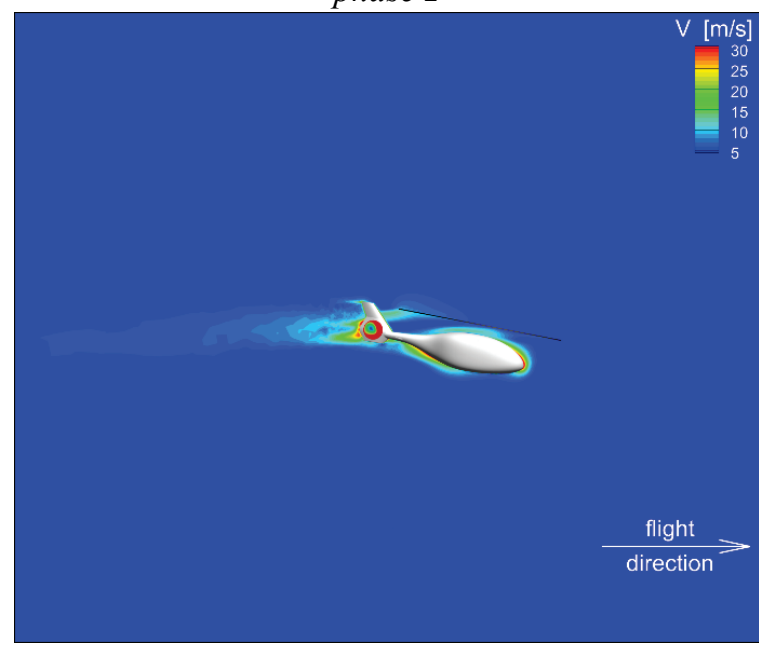

phase 4

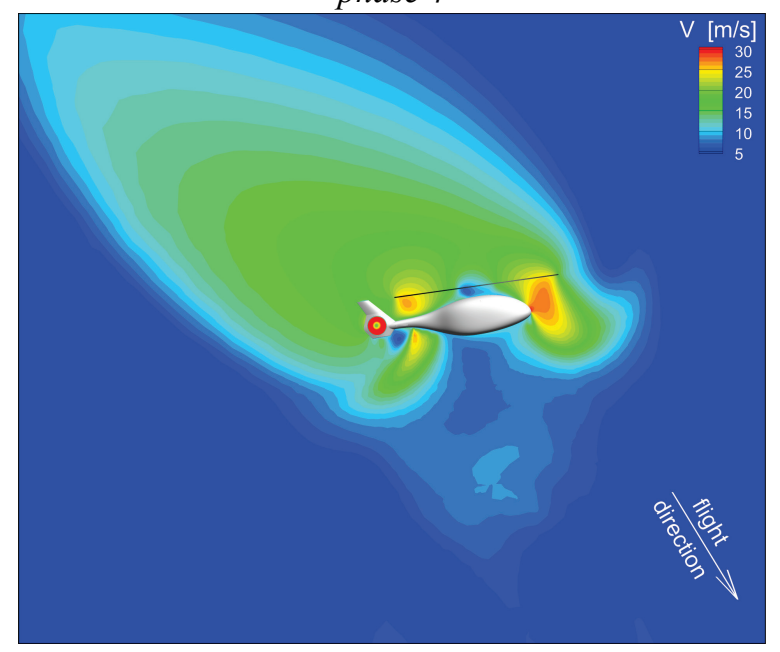

phase 6

Fig. 3. Velocity-magnitude contours around the helicopter in sequential phases (1-6) of flight 


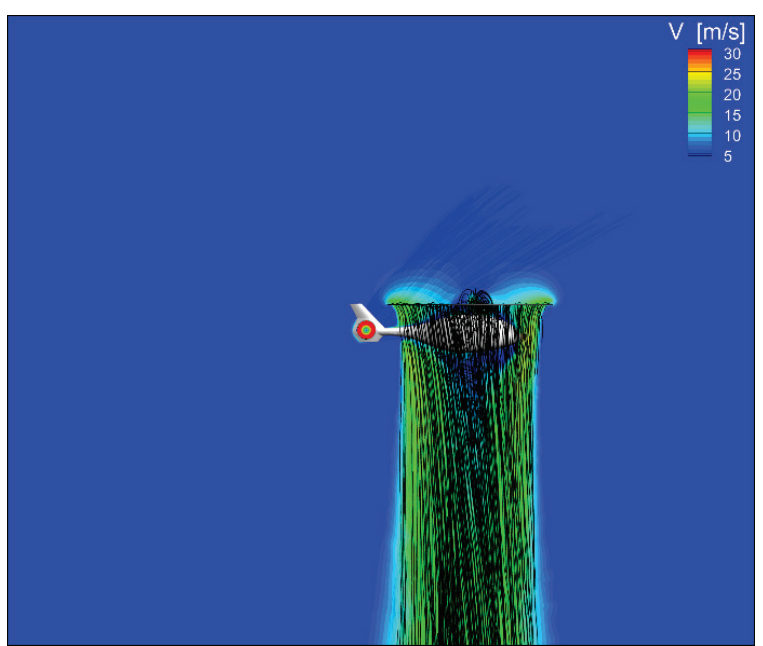

phase 1

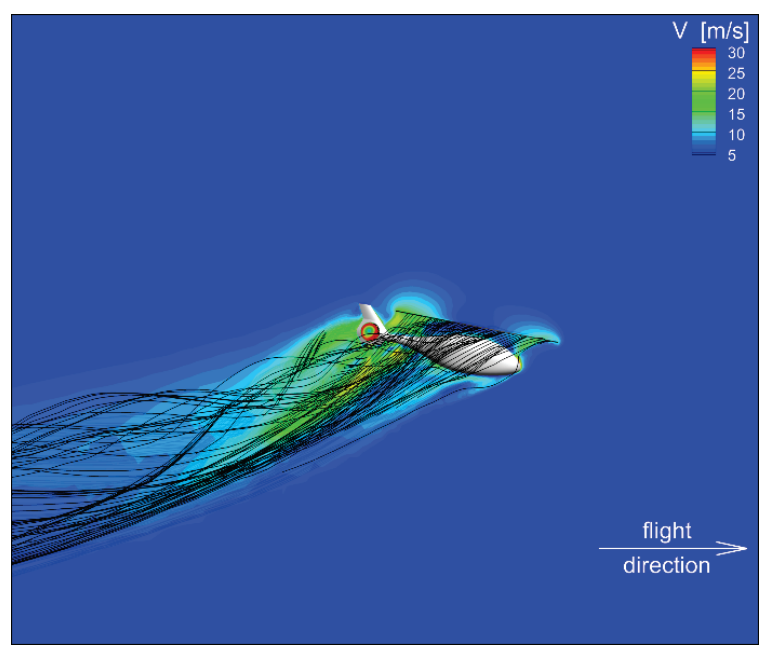

phase 3

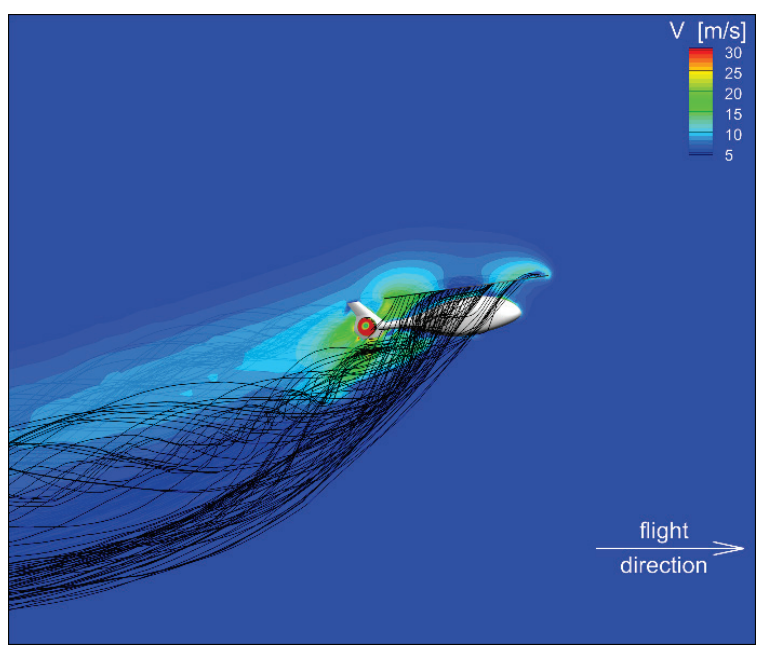

phase 5

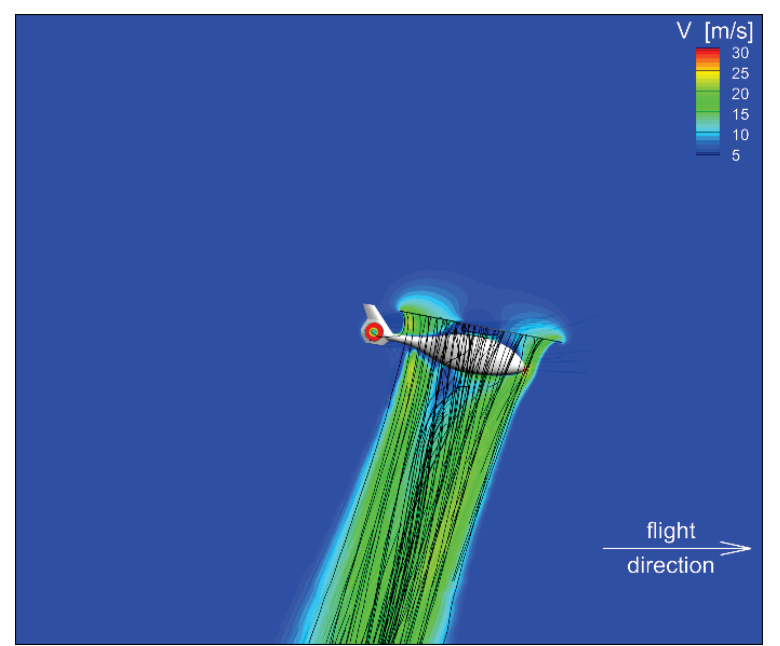

phase 2

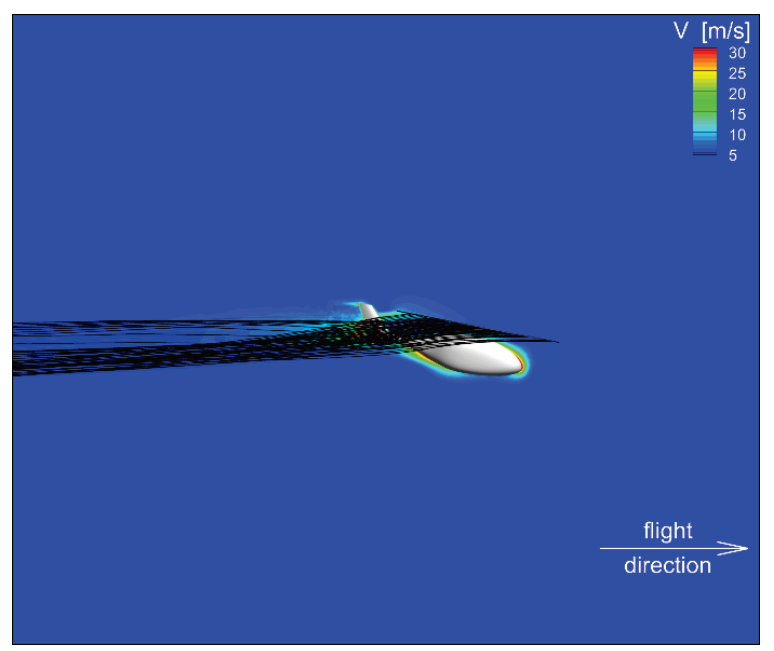

phase 4

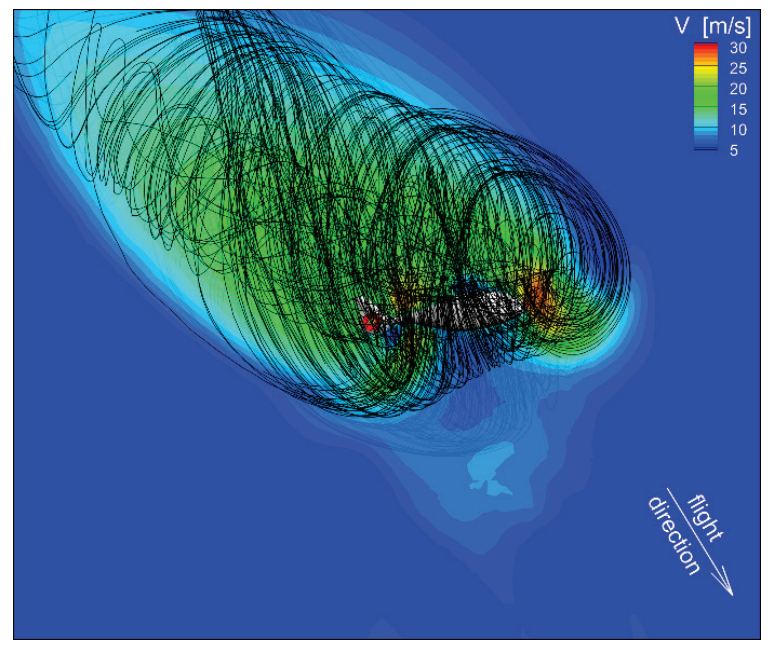

phase 6

Fig. 4. Streamlines flowing from the main and tail rotor, in sequential phases (1-6) of flight

Preliminary tests of the presented methodology confirm its high potential and suitability in rotorcraft research and development. This especially concerns the simulations of dangerous flight cases like emergency landing in autorotation or attempt to escape from the Vortex Ring State. Such simulations are planned as part of future applications of the developed methodology. 


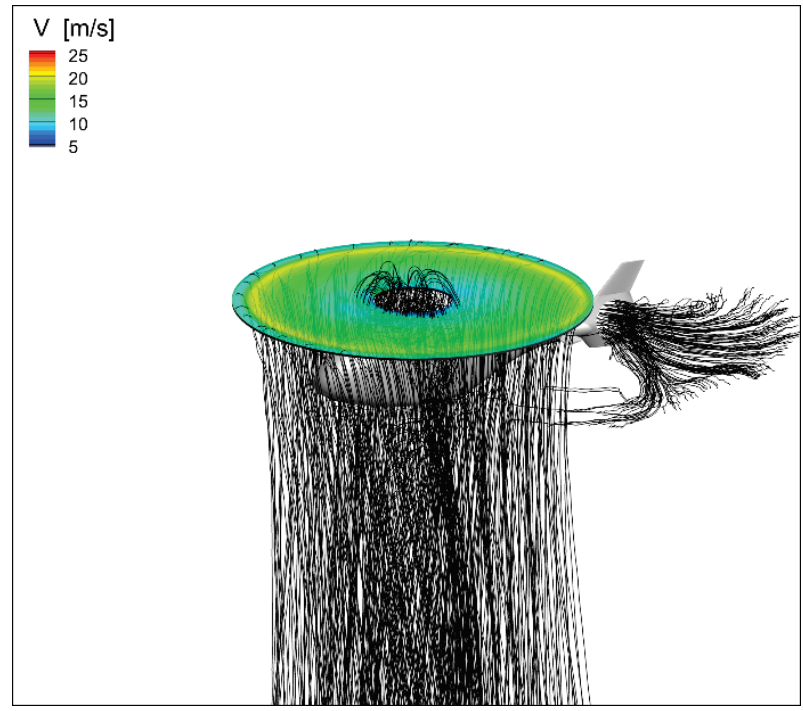

phase 1

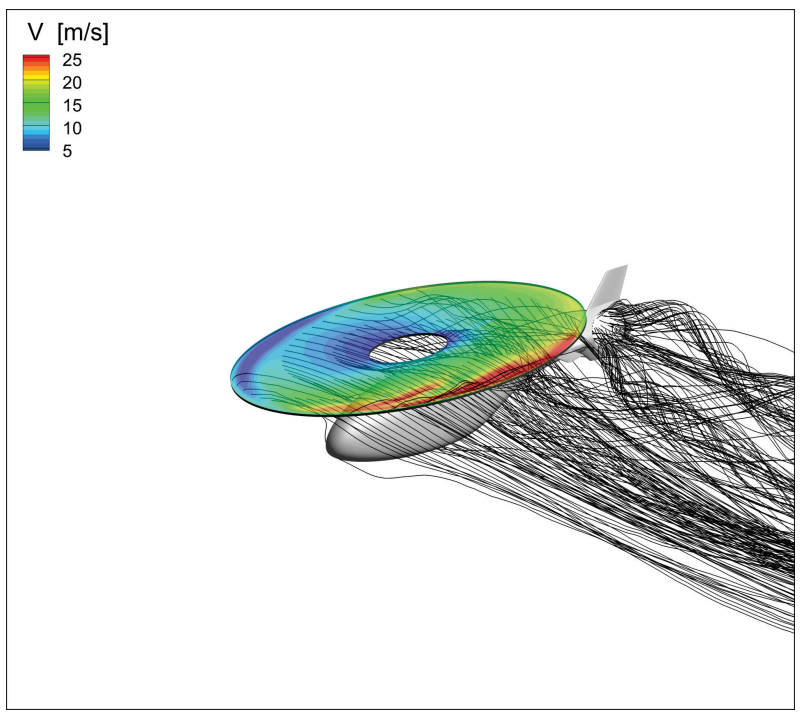

phase 3

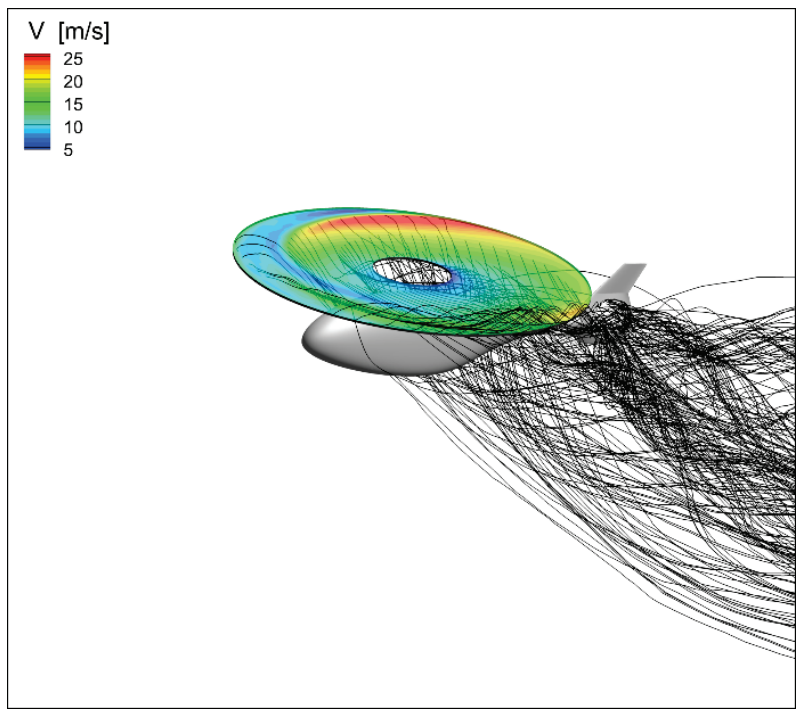

phase 5

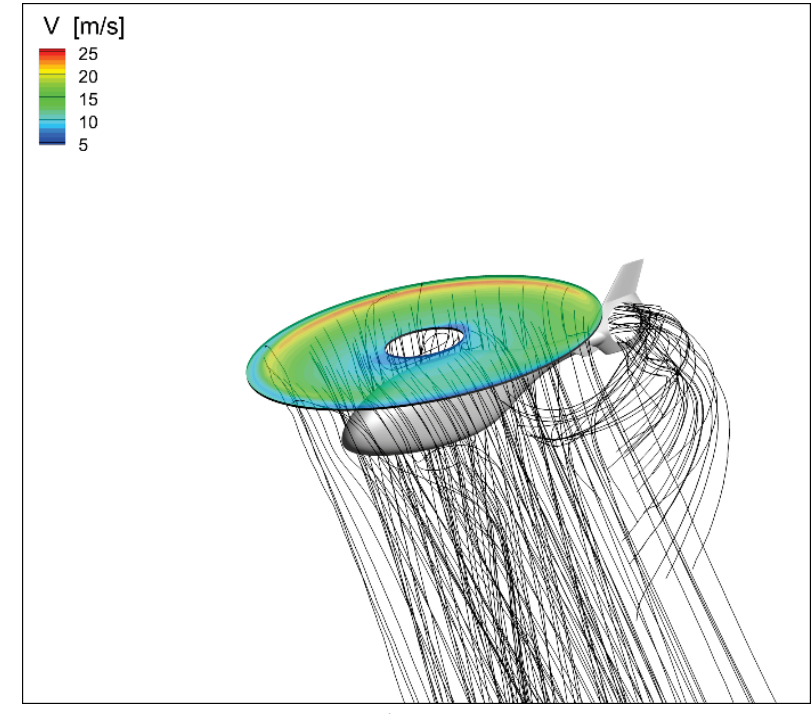

phase 2
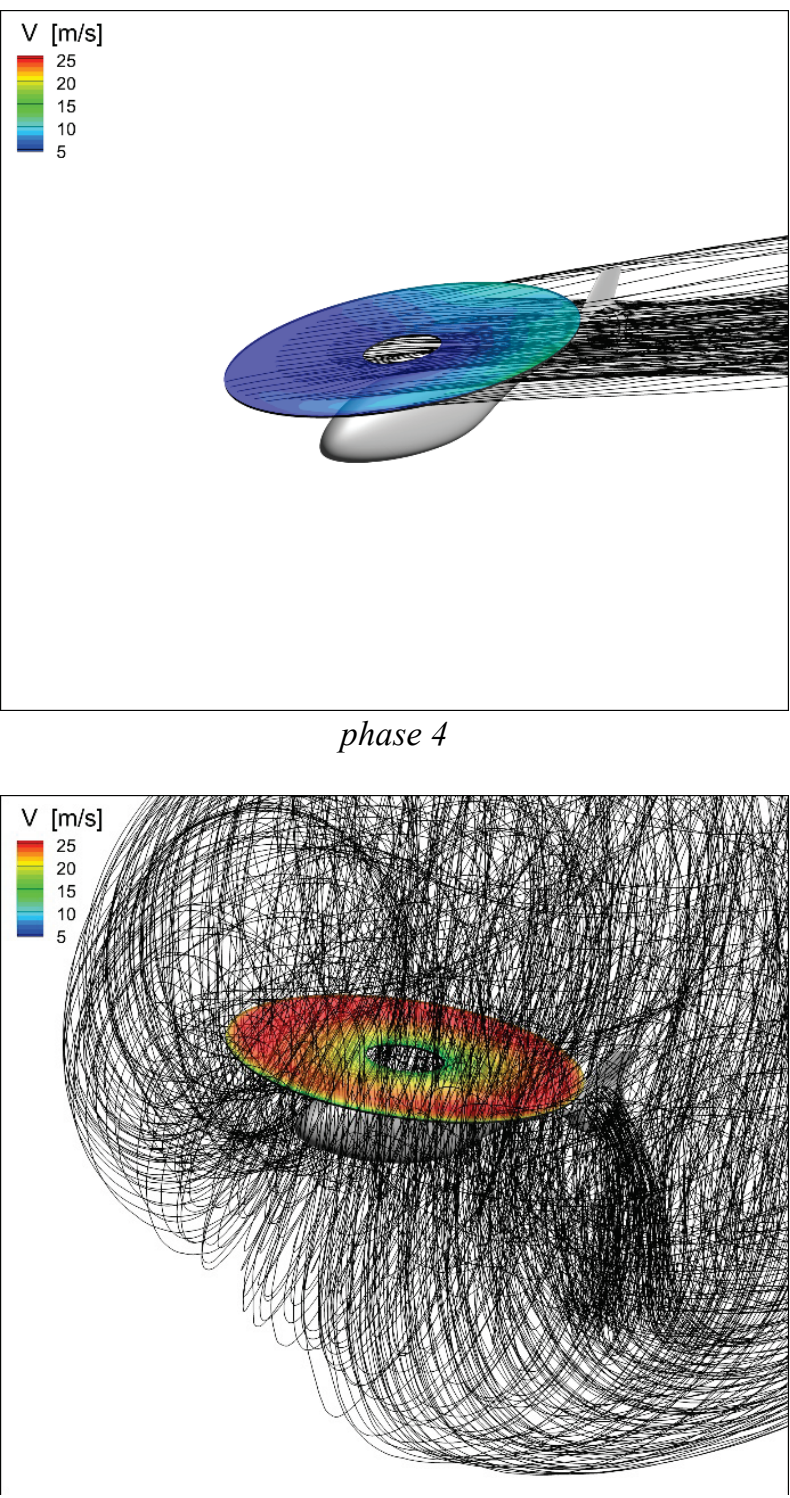

phase 6

Fig. 4. Velocity-magnitude contours on the main-rotor disc and streamlines flowing from the main and tail rotor in sequential phases (1-6) of flight 


\section{References}

[1] Inc., ANSYS FLUENT User's Guide. Release 18.2. Available from: http://www.ansys.com.

[2] Stalewski, W., Zoltak, J., Optimization of the Helicopter Fuselage with Simulation of Main and Tail Rotor Influence, In Proceedings of the 28th ICAS Congress of the International Council of the Aeronautical Sciences, ICAS, Brisbane, Australia, 23-28 September 2012.

[3] Stalewski, W., Simulation and Optimization of Control of Selected Phases of Gyroplane Flight, Special Issue "Selected Papers from the 7th International Conference on Experiments/ Process/System/Modelling/Simulation/Optimization (IC-EPSMSO 2017)", Computation, Vol. 6, Iss. 1, 2018.

Manuscript received 15 July 2019; approved for printing 23 September 2019 